\title{
Analysis of Different EOSs in Predicting the Ideal Curve and Deriving the Temperature Dependencies of Their Parameters
}

\author{
G. A. Parsafar ${ }^{1,2}$ and H. Saydi ${ }^{1}$
}

Received March 22, 2004

\begin{abstract}
The regularity shown by different fluids along the contour of the ideal compressibility factor $Z=P V /(R T)=1$ in the temperature-density plane is used to test the accuracy of different equations of state and derive temperature dependencies of their parameters. For a wide range of pure fluids, this contour, known as the Zeno line, has been empirically observed to be nearly linear. The precision of the van der Waals ( $\mathrm{vdW}$ ) equation in predicting the Zeno line has been evaluated and shown that this equation predicts a linear relation between temperature and density on the $Z=1$ contour, qualitatively. However, the line shows significant deviations from the experimental Zeno line. Experimental $P V T$ data for $\mathrm{CO}_{2}$ is used to obtain the temperature dependencies of the vdW parameters. The vdW equation with such temperature dependencies does not show a straight line for the $Z=1$ contour. This means that the equation is not able to predict the Zeno line, both qualitatively and quantitatively. Also, the accuracy of the modified vdW equations in predicting the Zeno line has been investigated. It is shown that none of these equations can predict the Zeno line qualitatively. However, the predicted line on the $Z=1$ contour given by some of these equations is near the experimental Zeno line. Assuming that the Zeno line must hold, the temperature dependence of the non-ideal thermal pressure, $A^{\prime \prime}$, of the linear isotherm regularity as $A^{\prime \prime}=a+b T+c / T$ has been derived. Such a temperature dependence was confirmed by experimental data. The derived expression for $A^{\prime \prime}$ was used to obtain the temperature dependence of the thermal pressure coefficient, which is in accordance with experimental data. Also, the temperature dependencies of the parameters of the dense system equation of state
\end{abstract}

\footnotetext{
${ }^{1}$ Department of Chemistry, Sharif University of Technology, P. O. Box 11365-9516, Tehran, Iran.

${ }^{2}$ To whom correspondence should be addressed. Email: parsafar@sharif.edu
} 
have been derived by imposing this regularity on it. The resulting expressions are in better agreement with experimental values than those previously obtained.

KEY WORDS: cubic equation of state; dense system equation of state; equation of state; linear isotherm regularity; Zeno line.

\section{INTRODUCTION}

Several empirical regularities have been reported for fluids; one which is related to the ideal curve $(Z=1)$ is the Zeno line, or the contour of $Z=1$, where the compressibility factor is the same as that for the ideal gas. The density of many fluids along the Zeno line has been found to be nearly a linear function of temperature. The ideal curves $(Z=1$ and its first derivatives) are useful as criteria for an assessment of the extrapolation behavior of simple substances, and also they contain important information on the behavior in the high-temperature, high-pressure region [1].

Although the linearity on the $Z=1$ contour was discovered by Batschinski [2] in 1906, it apparently had been forgotten until nearly six decades later. Beginning in the early 1960s, researchers investigated the Zeno line extensively [3] and it has been related to other thermodynamic properties. During late 1960s, Holleran [4-7] proposed several useful applications for the $Z=1$ contour. In recent years, from molecular dynamics simulations, Herschbach [8] obtained a Zeno line close to experimentally measured values over a wide range of densities by using the Lennard-Jones potential, the simple point charge (SPC), and the extended SPC (SPC/E) models for pure $\mathrm{H}_{2} \mathrm{O}$.

At low densities, the $Z=1$ contour is linear in a plot of $T$ versus $\rho$, where the zero-density intercept is the Boyle temperature. The most notable feature, however, is that the Zeno contour maintains its linearity even in the dense fluid region.

In this paper, first we have investigated the ability of different equations of state (including the vdW and eight other cubic equations, linear isotherm regularity, and dense system equation of state) in predicting the linearity on the $Z=1$ contour. Second, the possibility that temperature dependences of the parameters of the equations of state (EOSs) are chosen in such a way that (a) the Zeno line is kept linear and (b) the temperature dependencies are in accordance with experiment, has been examined. In the case of the linear isotherm regularity, the temperature dependence of $A^{\prime \prime}$ is obtained. The expression obtained for $A^{\prime \prime}$ is examined both directly, by using experimental data, and indirectly, by examining the temperature dependence of the thermal pressure coefficient, $\gamma=(\partial P / \partial T)_{\rho}$. 
Also, we have derived new functions for the temperature dependencies of the parameters of the dense system equation of state by imposing the Zeno line constraint on the EOS.

\section{ABILITY OF CUBIC EOSs TO PREDICT THE ZENO LINE}

The accuracy of an EOS may be tested via its ability to predict the well-known regularities, which have mostly been found experimentally. We investigate here the ability of the van der Waals (vdW) EOS in predicting the Zeno line. According to the Zeno line regularity, the density and temperature are linearly related to each other on the $Z=1$ contour as

$$
\frac{T}{T_{Z}}+\frac{\rho}{\rho_{Z}}=1,
$$

where $T_{Z}$ and $\rho_{z}$ are called the Zeno temperature and Zeno density, respectively, and $\rho=1 / v$ is the molar density. The $\mathrm{vdW}$ equation,

$$
\left(P+\frac{a}{v^{2}}\right)(v-b)=R T,
$$

when $Z=1$ the above equation may be written as

$$
\rho=-\frac{R T}{a}+\frac{1}{b},
$$

where $a$ and $b$ are the van der Waals parameters and $R$ is the gas constant. If $a$ and $b$ in Eq. (3) are substituted in terms of the critical variables $\left(b=v_{\mathrm{c}} / 3\right.$ and $\left.a=9 R v_{\mathrm{c}} T_{\mathrm{c}} / 8\right)$, the reduced form of this equation may be written as

$$
\frac{\rho}{\rho_{\mathrm{c}}}=3-\frac{8 T}{3 T_{\mathrm{c}}},
$$

where $\rho_{\mathrm{c}}$ and $T_{\mathrm{c}}$ are the critical density and temperature, respectively. However, we may substitute the parameters in term of the Boyle density, $\rho_{\mathrm{B}}$, and Boyle temperature, $T_{\mathrm{B}},\left(a=R T_{\mathrm{B}} / \rho_{\mathrm{B}}\right.$ and $\left.b=1 / \rho_{\mathrm{B}}\right)$ to reduce Eq. (3) to

$$
\frac{\rho}{\rho_{\mathrm{B}}}=1-\frac{T}{T_{\mathrm{B}}} .
$$

Therefore, the vdW EOS predicts a linear relation between temperature and density on the $Z=1$ contour, at least qualitatively, if $a$ and $b$ are constant. The experimental Zeno line for $\mathrm{CO}_{2}$ [8] is shown in Fig. 1, along with the lines predicted by Eqs. (4) and (5). As shown in this figure, the 


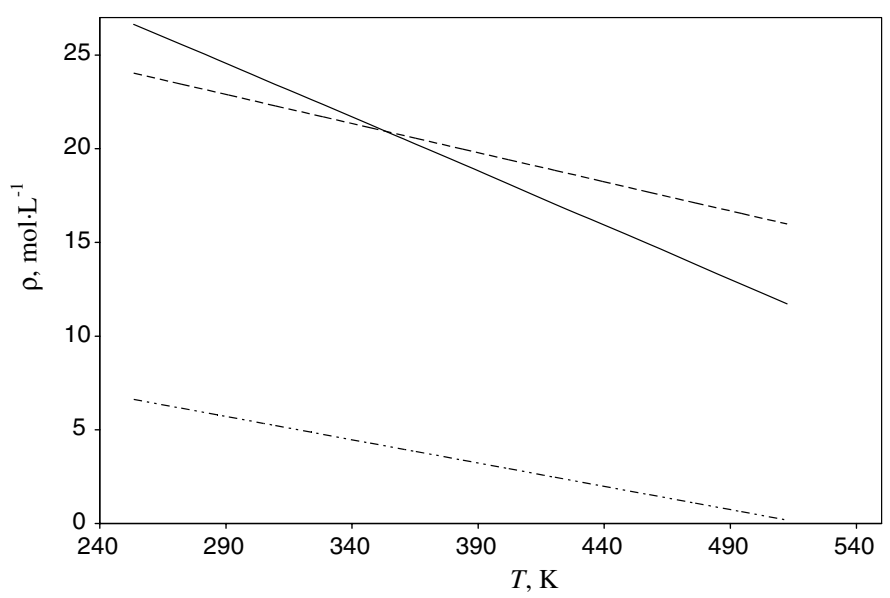

Fig. 1. Significant deviations of the lines given by Eqs. (4) (- - - ) and (5) (-.--) from the experimental Zeno line (-) for carbon dioxide $\left(\rho_{\mathrm{c}}=10.6249 \mathrm{~mol} \cdot \mathrm{L}^{-1}, T_{\mathrm{c}}=304.1282 \mathrm{~K}, \rho_{\mathrm{B}}=20.9740 \mathrm{~mol} \cdot \mathrm{L}^{-1}\right.$, and $T_{\mathrm{B}}=$ $716.554 \mathrm{~K})$.

predicted lines given by the vdW are significantly different from the experimental Zeno line of carbon dioxide, if the van der Waals parameters are temperature independent.

We may obtain the temperature dependencies of the $a$ and $b$ parameters in such a way that the EOS fits well onto experimental $P V T$ data. This is possible because the deviations of the calculated Zeno lines (Fig. 1) from the experimental line may be due to the temperature dependence of the parameters. To investigate such an expectation, we have used experimental $P V$ data of $\mathrm{CO}_{2}$ for different isotherms in a wide pressure range, along with the Solver in Excell, to find the values for the $a$ and $b$ parameters. The obtained results for the $b$ are well fitted with

$$
\frac{1}{b}=\frac{1}{b_{0}^{\prime}}+b_{1}^{\prime} T+\frac{b_{2}^{\prime}}{T}
$$

with a correlation coefficient of $R^{2}=0.999, b_{0}^{\prime}=3.152 \times 10^{-2} \mathrm{~L} \cdot \mathrm{mol}^{-1}$, $b_{1}^{\prime}=-1.159 \times 10^{-6} \mathrm{~mol} \cdot \mathrm{L}^{-1} \cdot \mathrm{K}^{-1}$, and $b_{2}^{\prime}=-1.240 \mathrm{~mol} \cdot \mathrm{K} \cdot \mathrm{L}^{-1}$. The obtained values for the $a$ parameter are well fitted with

$$
\frac{R T}{a}=a_{0}^{\prime}+a_{1}^{\prime} T+a_{2}^{\prime} T^{2}
$$

with a correlation coefficient of $R^{2}=0.999, a_{0}^{\prime}=2.0497 \mathrm{~mol} \cdot \mathrm{L}^{-1}, a_{1}^{\prime}=$ $-1.424 \times 10^{-3} \mathrm{~mol} \cdot \mathrm{L}^{-1} \cdot \mathrm{K}^{-1}$, and $a_{2}^{\prime}=-3.652 \times 10^{-8} \mathrm{~mol} \cdot \mathrm{L}^{-1} \cdot \mathrm{K}^{-2}$. These 


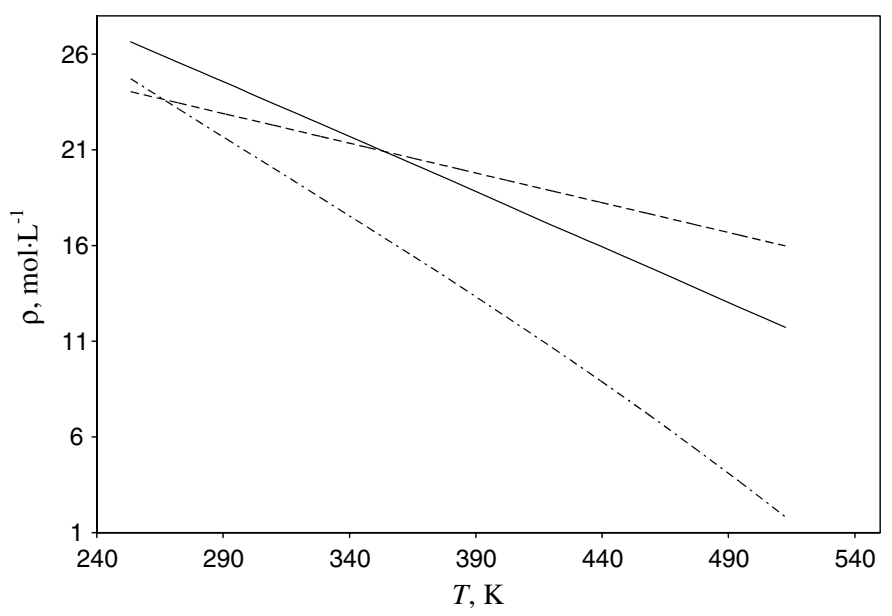

Fig. 2. Density versus temperature for the $Z=1$ contour, when the vdW parameters are temperature dependent (-.--) and temperature independent (- - - ) compared to the experimental Zeno line (-) for $\mathrm{CO}_{2}$.

temperature dependencies of the parameters are inserted into the vdW EOS. The predicted Zeno line of the resulting equation was then derived, which is shown in Fig. 2. As shown in this figure, the calculated Zeno line is closer to the experimental line at low temperatures, when temperature dependencies of the parameters are included. However, deviations from linearity can be observed when the parameters are temperature dependent as given by Eqs. (6) and (7). Therefore, we may conclude that the vdW EOS may not be able to predict the Zeno line both qualitatively and quantitatively. In a similar approach, one may derive a relation between $T$ and $\rho$ by using another EOS. The predicted relation is given for some modified vdW equations in Table I, although none of these equations give a linear relation between $\rho$ and $T$ on the Zeno contour.

We have made similar investigations on some cubic equations of state, including the Peng-Robinson (PR) [9], Soave-Redlich-Kwong (SRK) [10], Yu-Lu (YL) [11], Deiters (D) [12, 13], Patel-Teja (PT) [14], modified-Soave-Redlich-Kwong (MSRK) [15], Redlich-Kwong (RK) [16], and Soave-Peng-Robinson-Stryjek-Vera (SPRSV) [17]. The predicted $T$ versus $\rho$ on a $Z=1$ contour given by these EOSs are compared with the experimental Zeno line in Fig. $3 \mathrm{a}$ and $\mathrm{b}$ for $\mathrm{CO}_{2}$. As shown in these sub-figures, none of these EOSs gives a linear relation between $\rho$ and $T$ on the $Z=1$ contour. However, Deiters' EOS gives the best agreement. 
Table I. Obvious Nonlinear Expression Between $T_{\mathrm{r}}$ and $\rho_{\mathrm{r}}$ on the $Z=1$ Contour Given by Some Modified vdW EOSs $\left(\rho_{\mathrm{r}}=\rho / \rho_{\mathrm{B}}\right.$ and $\left.T_{\mathrm{r}}=T / T_{\mathrm{B}}\right)$

\begin{tabular}{llll}
\hline \multicolumn{1}{c}{ EOS } & $\begin{array}{l}\text { predicted relation } \\
\text { on } Z=1 \text { contour }\end{array}$ & $T_{\mathrm{B}}$ & $\rho_{\mathrm{B}}$ \\
\hline $\begin{array}{l}\text { Soave-Peng- } \\
\text { Robinson-Stryjek-Vera }\end{array}$ & $T_{\mathrm{r}}+2 T_{\mathrm{r}} \rho_{\mathrm{r}}-T_{\mathrm{r}} \rho_{\mathrm{r}}^{2}+\rho_{\mathrm{r}}=1$ & $a /(R b)$ & $1 / b$ \\
Redlich-Kwong & $T_{\mathrm{r}}^{1.5}+\frac{2}{3} T_{\mathrm{r}}^{1.5} \rho_{\mathrm{r}}+\frac{2}{3} \rho_{\mathrm{r}}=1$ & $(a /(R b))^{2 / 3}$ & $2 /(3 b)$ \\
Carnahan Starling & $\frac{8\left(8-\rho_{\mathrm{r}}\right)}{\left(4-\rho_{\mathrm{r}}\right)^{3}}=\frac{1}{T_{\mathrm{r}}}$ & $a /(R b)$ & $1 / b$ \\
\hline
\end{tabular}

\section{DERIVING THE TEMPERATURE DEPENDENCE OF THE ${ }^{\prime \prime}$ PARAMETER OF THE LINEAR ISOTHERM REGULARITY}

The linear isotherm regularity (LIR) may be written as $[18,19]$

$$
(Z-1) v^{2}=A+B \rho^{2},
$$

where $A$ and $B$ are temperature-dependent parameters that for pure fluids are given as

and

$$
A=A^{\prime \prime}-\frac{A^{\prime}}{R T}
$$

$$
B=\frac{B^{\prime}}{R T} .
$$

where $A^{\prime}$ and $B^{\prime}$ are constants, which are related to the attraction and repulsion terms of the average effective pair potential $[20,21]$ and $A^{\prime \prime}$ is related to the non-ideal thermal pressure.

Combination of Eq. (8) with $Z=1$ gives

$$
\rho_{r}^{2}=m T_{r}+n,
$$

where $\rho_{r}=\rho / \rho_{\mathrm{c}}, T_{\mathrm{r}}=T / T_{\mathrm{c}}$, and $m$ and $n$ are constant and are given as

$$
m=-\frac{R T_{\mathrm{c}} A^{\prime \prime}}{B^{\prime} \rho_{\mathrm{c}}^{2}}
$$

and

$$
n=\frac{A^{\prime}}{B^{\prime} \rho_{\mathrm{c}}^{2}} .
$$



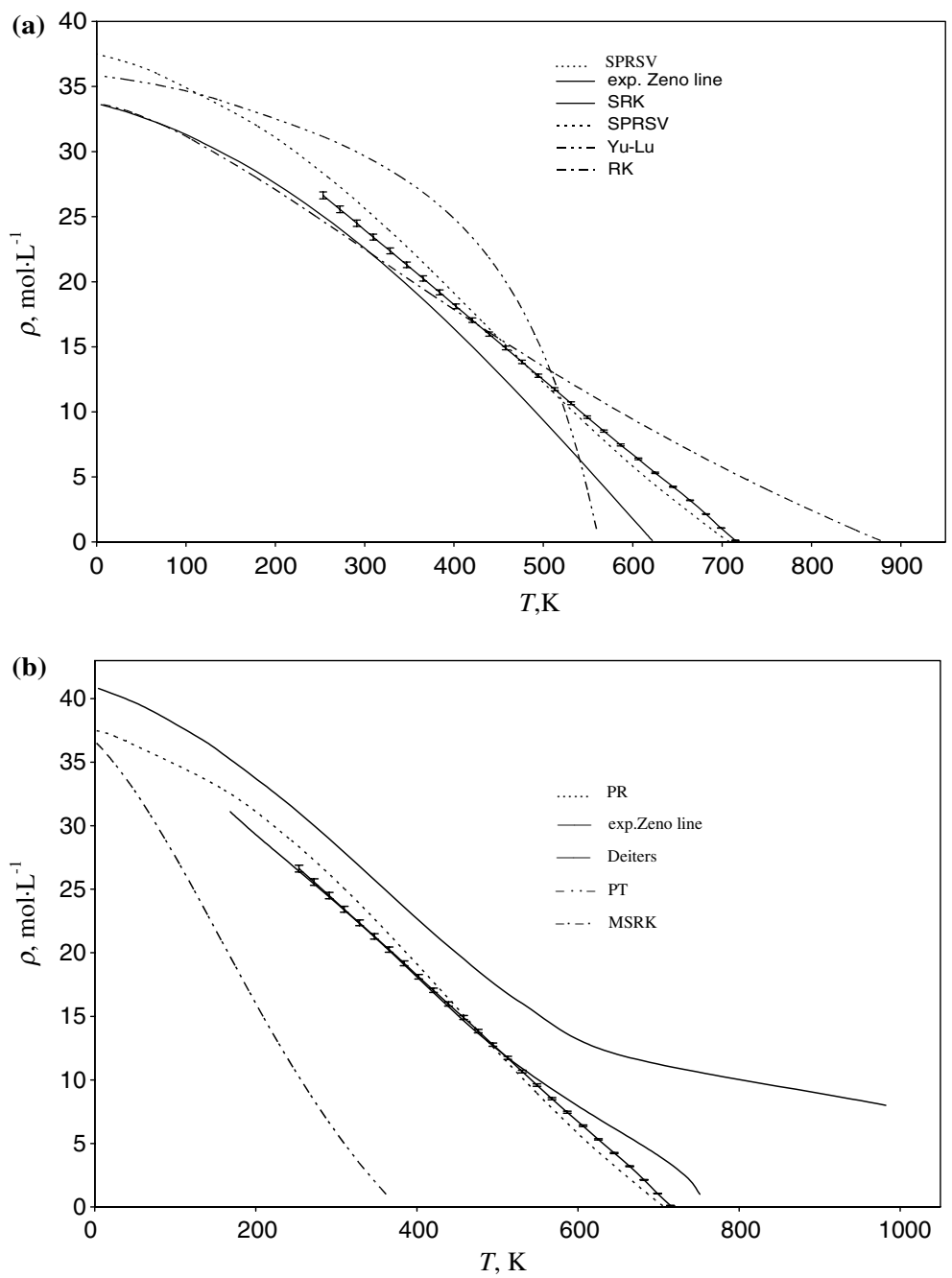

Fig. 3. Predicted $T$ versus $\rho$ given by (a) SRK, SPRSV, Yu-Lu, and RK; (b) PT, PR, Deiters, and MSRK equations of state compared with the experimental Zeno line for carbon dioxide.

Therefore, the LIR in its present formulation does not predict a linear relation between temperature and density on the $Z=1$ contour, if $A^{\prime \prime}$ is considered to be a constant.

The Zeno line may be written as

$$
\rho_{\mathrm{r}}=\alpha+\beta T_{\mathrm{r}},
$$


where $\alpha$ and $\beta$ are as follows:

$$
\begin{aligned}
& \alpha=\frac{\rho_{\mathrm{z}}}{\rho_{\mathrm{c}}}, \\
& \beta=-\frac{\rho_{\mathrm{z}} T_{\mathrm{c}}}{\rho_{\mathrm{c}} T_{\mathrm{Z}}},
\end{aligned}
$$

where $T_{\mathrm{Z}}$ and $\rho_{\mathrm{Z}}$ are the intercepts of temperature and density in the $T-\rho$ plane. Consequently, inserting Eq. (14) into Eq. (11) yields

$$
A^{\prime \prime}+\frac{2 \alpha \beta B^{\prime}}{R}+\left(\frac{\alpha^{2} B^{\prime}}{R}-\frac{A^{\prime}}{R}\right) \frac{1}{T_{\mathrm{r}}}+\frac{\beta^{2} B^{\prime}}{R} T_{\mathrm{r}}=0 .
$$

Since $A^{\prime \prime}$ is related to the thermal pressure coefficient $(\partial P / \partial T)_{\rho}[20]$ which is obviously temperature dependent, one may expect that it is temperature dependent as well. We may derive the temperature dependence of the $A^{\prime \prime}$ parameter by solving Eq. (17) for $A^{\prime \prime}$. The derived expression for $A^{\prime \prime}$ leads to a linear relation between $\rho$ and $T$ on the $Z=1$ contour according to the LIR. From Eq. (17), we find the temperature dependence of $A^{\prime \prime}$ as

$$
A^{\prime \prime}=a+b T_{\mathrm{r}}+\frac{c}{T_{\mathrm{r}}}
$$

where $a=-2 \alpha \beta B^{\prime} / R, b=-\beta^{2} B^{\prime} / R$, and $c=A^{\prime} / R-\alpha^{2} B^{\prime} / R$, and all are constant parameters that depend on the selected fluid.

We may investigate the accuracy of Eq. (18) by using experimental data. The value of $A^{\prime \prime}$ was calculated at different temperatures by using experimental $P V T$ data for argon and carbon dioxide [22] as follows: we may plot $(Z-1) v_{\mathrm{r}}^{2}$ versus $\rho_{\mathrm{r}}^{2}$ for an isotherm for temperatures and densities for which the LIR is valid $\left(\rho>\rho_{\mathrm{B}}\right.$ and $\left.T \leqslant 2 T_{\mathrm{B}}\right)$. The values of $A$ and $B$ may be obtained from the intercept and slope of the line at the temperature for which the isotherm is plotted. The temperature range was divided into $20 \mathrm{~K}$ intervals, for which $A^{\prime \prime}$ has been shown to be constant [20]. Then, $A$ is plotted versus $1 / T$ for each interval to obtain $A^{\prime \prime}$ for its intermediate temperature. The calculated results are plotted in Fig. 4 for $\mathrm{CO}_{2}$ and Ar which are fitted well with Eq. (18).

The $A^{\prime \prime}$ parameter of the LIR is related to the non-ideal thermal pressure as [18]

$$
A^{\prime \prime}=\frac{1}{\rho^{2}}\left(\frac{\gamma}{R \rho}-1\right),
$$




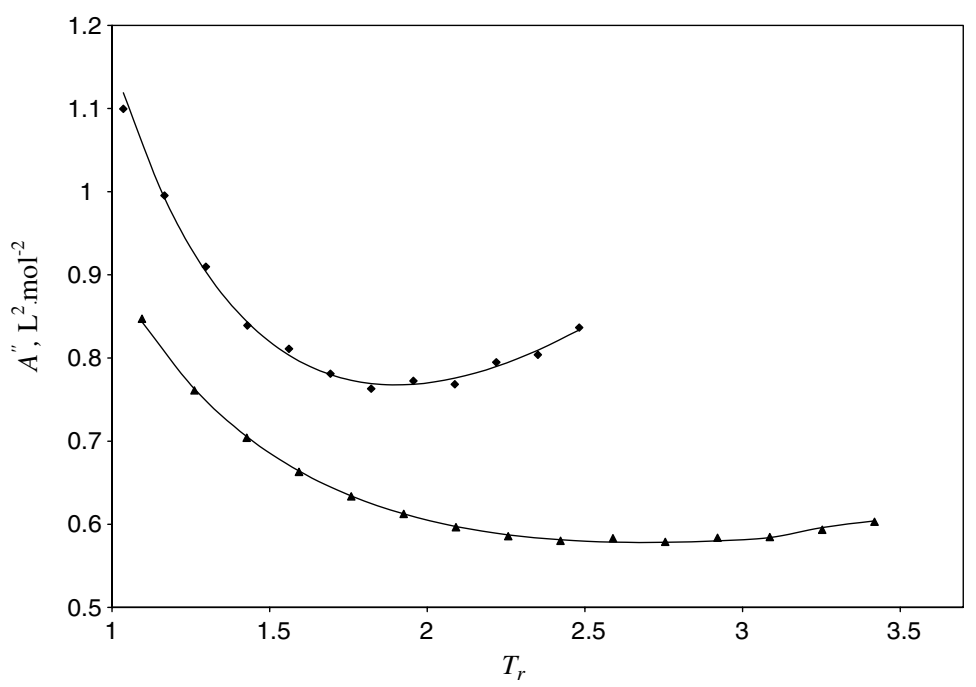

Fig. 4. Experimental values of $A^{\prime \prime}$ which are well fitted with Eq. (18) for carbon dioxide (ם) and $\operatorname{argon}(\boldsymbol{\Delta})$.

where $\gamma=(\partial P / \partial T)_{v}$ is the thermal pressure coefficient and $R$ is the gas constant. If $A^{\prime \prime}$ is substituted from Eq. (18), then the temperature dependence of $\gamma$ can be obtained for each isochore as

$$
\gamma=c_{0}^{\prime}+c_{1}^{\prime} T_{\mathrm{r}}+\frac{c_{2}^{\prime}}{T_{\mathrm{r}}}
$$

where $c_{0}^{\prime}, c_{1}^{\prime}$, and $c_{2}^{\prime}$ are density-dependent parameters. We may obtain the temperature dependence of the thermal pressure coefficient by using the empirical EOS reported for different fluids ( $\mathrm{Ar}\left[23,24\right.$ ], $\mathrm{CO}_{2}$ [25], $\mathrm{N}_{2}$ [26, 27], and $\mathrm{O}_{2}$ [28]). The values obtained for $\gamma$ from the empirical EOSs are fitted with Eq. (20) as shown in Fig. 5. This figure shows that the values of $\gamma$ are fitted well with Eq. (20) for four different fluids.

\section{USING THE ZENO LINE TO DERIVE THE TEMPERATURE DEPENDENCIES OF THE DENSE SYSTEM EQUATION OF STATE PARAMETERS}

The dense system equation of state (DSEOS) is derived as [29]

$$
P_{\mathrm{r}} v_{\mathrm{r}}^{2}=A_{0}+A_{1} \rho_{\mathrm{r}}+A_{2} \rho_{\mathrm{r}}^{2}
$$



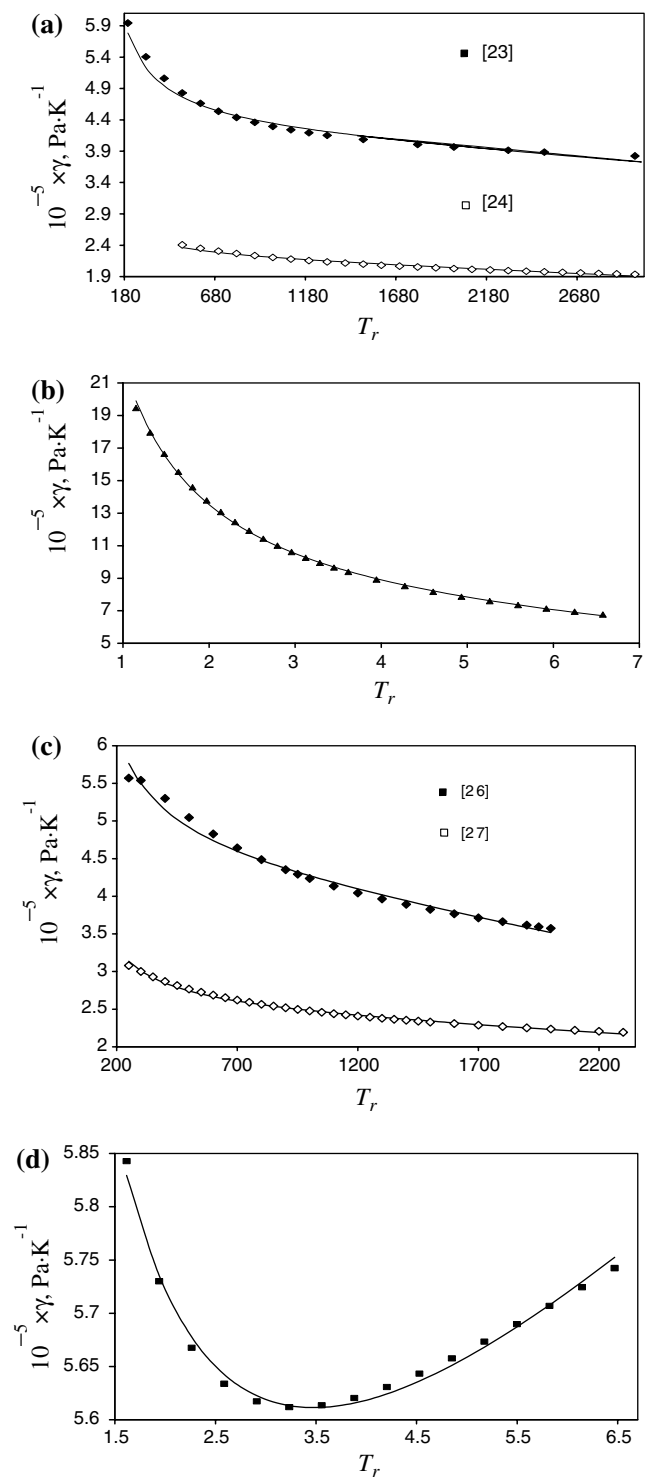

Fig. 5. Empirical values of $\gamma=(\partial P / \partial T)_{\rho}$ which are well fitted in Eq. (20) for (a) argon; (b) carbon dioxide; (c) nitrogen; (d) oxygen. 
where $\rho_{\mathrm{r}}=\rho / \rho_{\mathrm{c}}, v_{\mathrm{r}}=v / v_{\mathrm{c}}$, and $P_{\mathrm{r}}=P / P_{\mathrm{c}}$ are dimensionless variables. On the basis of the linear dependence of the isochoric heat capacity with temperature, the $A_{0}, A_{1}$, and $A_{2}$ parameters were derived as [29]

$$
A_{i}(T)=a_{i}+b_{i} T+c_{i} T^{2}-d_{i} T \ln T
$$

for which $a_{i}, b_{i}, c_{i}$, and $d_{i}$ are constant and depend on the fluid $(i=0,1$, and 2).

The mathematical form of the DSEOS on the $Z=1$ contour is obtained as

$$
\frac{R T}{P_{\mathrm{c}} V_{\mathrm{c}}}=A_{0} \rho_{\mathrm{r}}+A_{1} \rho_{\mathrm{r}}^{2}+A_{2} \rho_{\mathrm{r}}^{3} .
$$

When $\rho_{\mathrm{r}}=\alpha+\beta T$ is inserted into Eq. (23), we find that

$$
\begin{aligned}
& \left(\alpha A_{0}+\alpha^{2} A_{1}+\alpha^{3} A_{2}\right)+\left(\beta A_{0}+2 \alpha \beta A_{1}+3 \alpha^{2} \beta A_{2}-\frac{R}{P_{\mathrm{c}} V_{\mathrm{c}}}\right) T \\
& \quad+\left(A_{1} \beta^{2}+3 \alpha \beta^{2} A_{2}\right) T^{2}+\beta^{3} A_{2} T^{3}=0 .
\end{aligned}
$$

If the temperature dependencies of the $A_{i}$ s are taken into account, according to Eq. (22), and $\ln T$ is expanded about an arbitrary non-zero value, then Eq. (24) reduces to a power series in terms of temperature.

The DSEOS can predict the Zeno line when each coefficient of the series is zero. Such conditions are satisfied only when the parameters $d_{0}, d_{1}, d_{2}$, and $c_{2}$ are all made zero. Hence, we may conclude that

$$
\begin{aligned}
& A_{0}=a_{0}+b_{0} T+c_{0} T^{2}, \\
& A_{1}=a_{1}+b_{1} T+c_{1} T^{2}, \\
& A_{2}=a_{2}+b_{2} T .
\end{aligned}
$$

However, the temperature dependencies of the experimental values of the $A_{i}$ s are quite different from those given by Eqs. (25)-(27); for example, see Fig. 5 in Ref. 29. Therefore, we may conclude that the temperature dependencies of the $A_{i}$ s given by Eq. (22) are not consistent with the Zeno line. For this reason we have proposed new expressions for the temperature dependencies of the $A_{i} \mathrm{~s}$, in such a way that the Zeno line holds, at least qualitatively. The DSEOS can predict the Zeno line when the $A_{i}$ s are given as

$$
\begin{aligned}
A_{i}= & \frac{a_{-i n}}{T^{n}}+\frac{a_{-i n+1}}{T^{n-1}}+\cdots+a_{i 0}+a_{i 1} T+\cdots+a_{i n} T^{n} \\
& +\cdots+a_{(i n-i+2)} T^{n-i+2} .
\end{aligned}
$$


If $A_{i}$ 's are substituted from Eq. (28) into Eq. (24), then the resulting equation is equal to zero if

$$
\begin{aligned}
& A_{2}=\frac{a_{-2 n}}{T^{n}}+\frac{a_{-2(n-1)}}{T^{n-1}}+\cdots+a_{20}+a_{21} T+\cdots+a_{2 n} T^{n}, \\
& A_{1}=\frac{a_{-1 n}}{T^{n}}+\frac{a_{-1(n-1)}}{T^{n-1}}+\cdots+a_{10}+a_{11} T+\cdots+a_{1(n+1)} T^{n+1}, \\
& A_{0}=\frac{a_{-0 n}}{T^{n}}+\frac{a_{-0(n-1)}}{T^{n-1}}+\cdots+a_{00}+a_{01} T+\cdots+a_{0(n+2)} T^{n+2} .
\end{aligned}
$$

We have to choose a value for $n$ in such a way that the temperature dependencies of the $A_{i}$ 's are in accordance with experiment. For $n=0$ and $n=1$, we found that the expressions for the $A_{i}$ 's are not in agreement with experiment. Therefore, when we assume that $n=2$, then we will compare the results with experiment. For such a value for $n$, Eqs. (29)-(31) are reduced to

$$
\begin{aligned}
& A_{2}=\frac{a_{2}}{T^{2}}+\frac{b_{2}}{T}+c_{2}+d_{2} T+e_{2} T^{2}, \\
& A_{1}=\frac{a_{1}}{T^{2}}+\frac{b_{1}}{T}+c_{1}+d_{1} T+e_{1} T^{2}+f_{1} T^{3}, \\
& A_{0}=\frac{a_{0}}{T^{2}}+\frac{b_{0}}{T}+c_{0}+d_{0} T+e_{0} T^{2}+f_{0} T^{3}+g_{0} T^{4} .
\end{aligned}
$$

The experimental values of the $A_{i}$ 's for Ar were fitted into Eqs. (32)-(34), which are shown in Fig. 6. Values of the parameters of Eqs. (32)-(34) are given in Table II. The experimental values of the $A_{i}$ 's (for Ar) were also fitted with the originally proposed expression, Eq. (22). As shown in this figure, our expressions are in better agreement with the experimental values than the original ones.

\section{CONCLUSION}

We have evaluated the accuracy of the vdW EOS equation for predicting the Zeno line and have shown that this equation predicts a linear relationship between temperature and density on the $Z=1$ contour. However, the predicted line has significant deviation from the experimental Zeno line (see Fig. 1).

To find a reason for such a deviation, we have used $P V T$ data of $\mathrm{CO}_{2}$ to find temperature dependencies of the $\mathrm{vdW}$ parameters. The predicted line was found to be in better agreement with the experimental Zeno line at low temperatures, when temperature dependencies of the parameters were taken into account. However, the predicted line shows a noticeable 

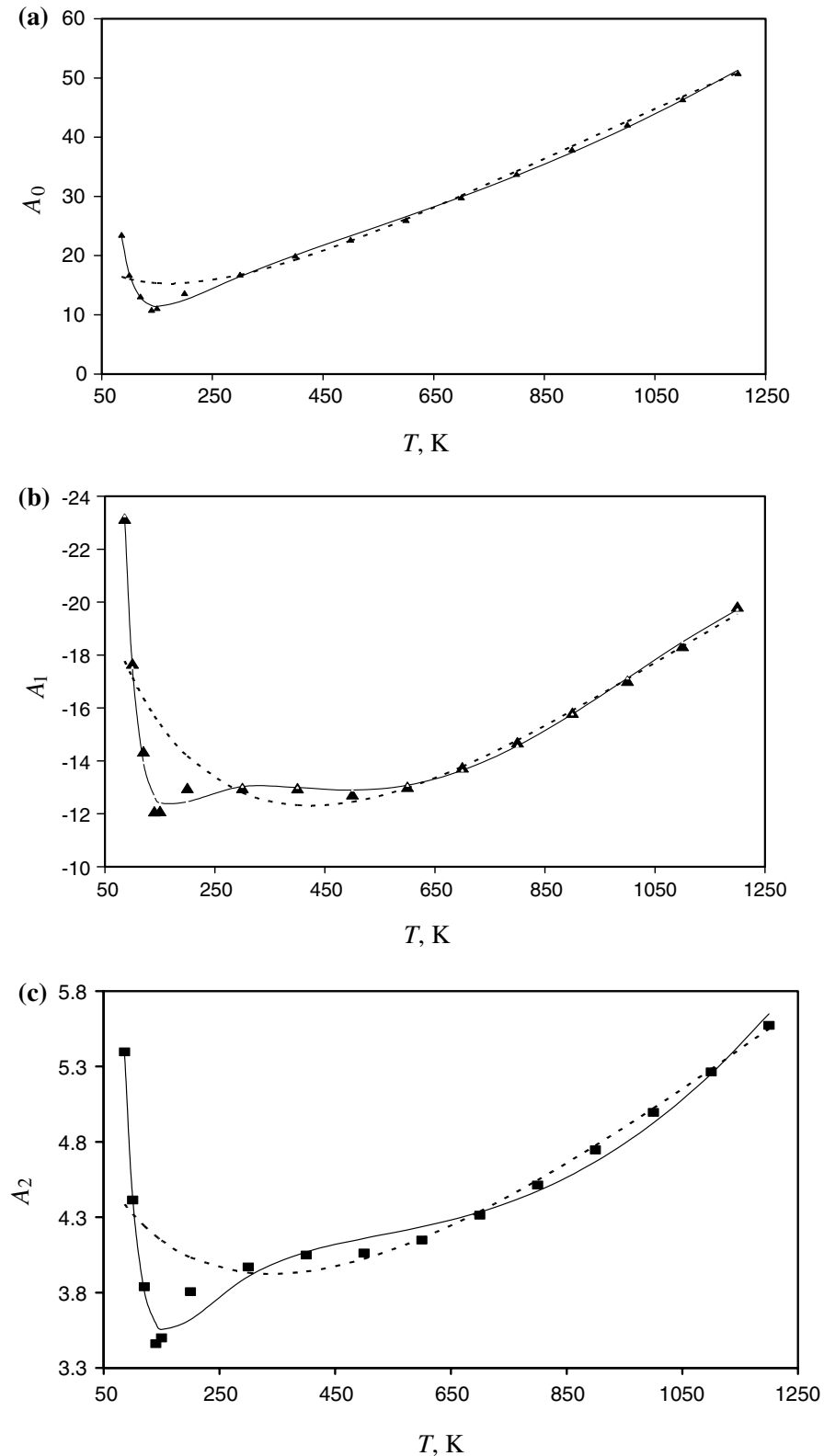

Fig. 6. Fitted the experimental values of (a) $A_{0}$ into Eqs. (32) ( - ) and (22) (- - - - -); (b) $A_{1}$ into Eqs. (33) (-) and (22) (- - - - -); (c) $A_{2}$ in Eqs. (34) ( $($ ) and (22) (- . . - ) for Ar. 
Table II. Values of the Parameters of Eqs. (32)-(34) for Argon

\begin{tabular}{lc}
\hline Parameter & Value \\
\hline$a_{0}\left(\mathrm{~K}^{2}\right)$ & 67595 \\
$b_{0}(\mathrm{~K})$ & -11134 \\
$c_{0}$ & 72.249 \\
$d_{0}\left(\mathrm{~K}^{-1}\right)$ & -0.13960 \\
$e_{0}\left(\mathrm{~K}^{-2}\right)$ & $2.1197 \times 10^{-4}$ \\
$f_{0}\left(\mathrm{~K}^{-3}\right)$ & $-1.1682 \times 10^{-7}$ \\
$g_{0}\left(\mathrm{~K}^{-4}\right)$ & $2.4837 \times 10^{-11}$ \\
$a_{1}\left(\mathrm{~K}^{2}\right)$ & -48993 \\
$b_{1}(\mathrm{~K})$ & 7776.3348 \\
$c_{1}$ & -55.822 \\
$d_{1}\left(\mathrm{~K}^{-1}\right)$ & 0.099900 \\
$e_{1}\left(\mathrm{~K}^{-2}\right)$ & $-1.0006 \times 10^{-4}$ \\
$f_{1}\left(\mathrm{~K}^{-3}\right)$ & $3.1032 \times 10^{-4}$ \\
$a_{2}\left(\mathrm{~K}^{2}\right)$ & 8.1436 \\
$b_{2}(\mathrm{~K})$ & $-6.5040 \times 10^{-3}$ \\
$c_{2}$ & $4.2582 \times 10^{-6}$ \\
$d_{2}\left(\mathrm{~K}^{-1}\right)$ & 1044.9 \\
$e_{2}\left(\mathrm{~K}^{-2}\right)$ & 73303 \\
\hline
\end{tabular}

curvature in the latter case (see Fig. 2). Therefore, we have concluded that the vdW EOS is unable to predict the Zeno line both quantitatively and qualitatively, even when the temperature dependencies of its parameters are included. Additionally, the accuracy of some well known modified vdW EOSs in predicting the Zeno line has been investigated (see Table I). It was shown that none of these EOSs predicts the Zeno line, even qualitatively.

The accuracy of eight different cubic EOSs in predicting the Zeno line was investigated numerically in this work. Among these EOSs, the Deiters' equation shows the best agreement with the experimental Zeno line (see Fig. 3). We have forced the LIR regularity in such a way that it satisfies the Zeno line regularity, quantitatively, from which the temperature dependence of the $A^{\prime \prime}$ parameter was obtained for the first time. Such a dependency was confirmed by experimental data (see Fig. 4). The derived expression for the $A^{\prime \prime}$ was used to obtain the temperature dependence of the thermal pressure coefficient, which was found to be in accordance with experimental data (see Fig. 5).

The same application was also applied to the DSEOS to find temperature dependencies of its parameters. The derived expressions were found to be in better agreement with experiment than those previously proposed 


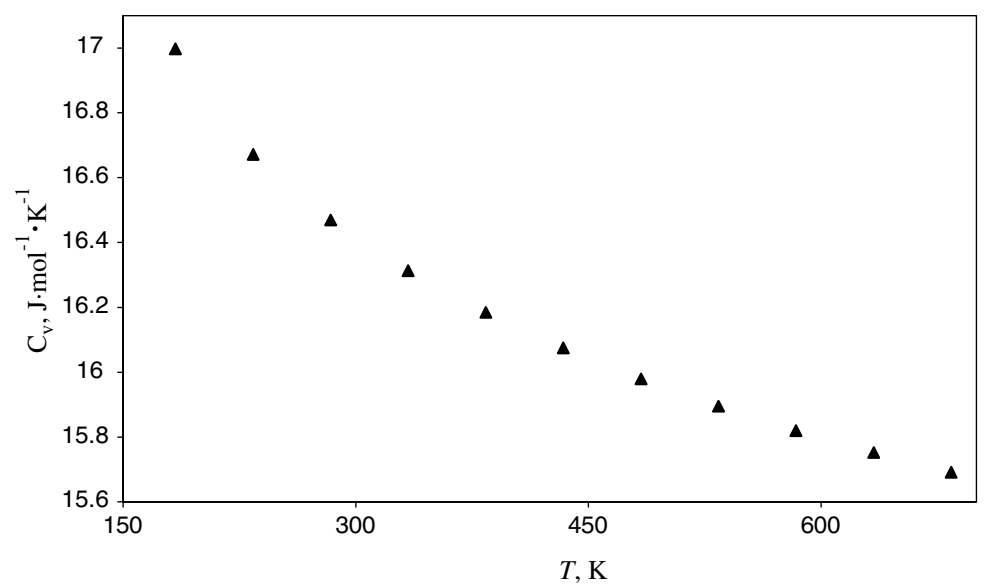

Fig. 7. Significant deviation of $C_{\mathrm{V}}$ versus $T$ from a straight line over a wide temperature range for $\operatorname{argon}\left(\rho=28 \mathrm{~mol} \cdot \mathrm{L}^{-1}\right)$.

(see Fig. 6). The reason for such better agreement may be due to the limited assumption used in the original work, in which the isochoric heat capacity $\left(C_{v}\right)$ was assumed to be linear with temperature. The empirical values of $C_{v}$ for $\operatorname{Ar}[22]$ versus temperature are shown in Fig. 7 for $\rho=28 \mathrm{~mol} \cdot \mathrm{L}^{-1}$. The linearity is reasonable for a short temperature range only.

\section{ACKNOWLEDGMENT}

We wish to acknowledge the Sharif University of Technology Research Council for financial support.

\section{REFERENCES}

1. R. Span and W. Wagner, Int. J. Thermophys. 18:1415 (1997).

2. A. Batschinski, Ann. Phys. 19:307 (1906).

3. T. E. Morsy, Doctoral Dissertation, University of Karlsruhe, Karlsruhe, Germany (1963).

4. M. Holleran, J. Phys. Chem. 72:1230 (1968).

5. M. Holleran, J. Phys. Chem. 73:167 (1969).

6. M. Holleran, Ind. Eng. Chem. Res. 29:632 (1990).

7. M. Holleran, J. Chem. Phys. 49:39 (1968).

8. R. Herschbach, J. Phys. Chem. 96:2307 (1992).

9. D. Y. Peng and D. B. Robinson, Ind. Eng. Chem. Fundam. 15:59 (1976).

10. G. Soave, Chem. Eng. Sci. 27:1197 (1972).

11. M.Yu and C.-Y. Lu, Fluid Phase Equilib. 34:1 (1987).

12. D. Deiters, Chem. Eng. Sci., 36:1139 (1981).

13. V. G. Boanza, M. Caceres, and J. Nunez, Fluid Phase Equilib. 78:43 (1992).

14. C. Patel and A. S. Teja, Chem. Eng. Sci. 37:463 (1982). 
15. J. O. Valderrama, H. L. Puente, and A. A. Ibrahim, Fluid Phase Equilib. 34:1 (1987).

16. O. Redlich and J. N. S. Kwong, Chem. Rev. 44:233 (1949).

17. R. Stryjek and J. H. Vera, Can. J. Chem. Eng. 64:334 (1986).

18. G. A. Parsafar and E. A. Mason, J. Phys. Chem. 97:9048 (1993).

19. G. A. Parsafar, J. Sci. Islamic Repub. Iran 2:111 (1991).

20. G. A. Parsafar, F. Kermanpour and B. Najafi, J. Phys. Chem. 103:B (1999).

21. G. A. Parsafar and F. Kermanpour, Int. J. Thermophys. 22:1795 (2001).

22. http://webbook.nist.gov/chemistry/fluid.

23. R. B. Stewart and R. T Jacobsen, J. Phys. Chem. Ref. Data 18:639 (1989).

24. Ch. Tageler, R. Span, and W. Wagner, J. Phys. Chem. Ref. Data 28:779 (1999).

25. R. Span and W. Wagner, J. Phys. Chem. Ref. Data 25:1509 (1996).

26. R. T. Jacobsen, R. B. Stewart, and M. Jahangiri, J. Phys. Chem. Ref. Data 15:735 (1986).

27. R. Span, E. W. Lemmon, R T. Jacobsen, W. Wagner, and A. A. Yokozeki, J. Phys. Chem. Ref. Data 29:1361 (2000).

28. R. B. Stewart, R. T Jacobsen, and W. Wagner, J. Phys. Chem. Ref. Data 20:917 (1991).

29. G. A. Parsafar, N. Farzi, and B. Najafi, Int. J. Thermophys. 18:917 (1997). 